\title{
1 Flotation of alumina on the surface of 2 the electrolyte in an aluminum 3 electrolysis cell
}

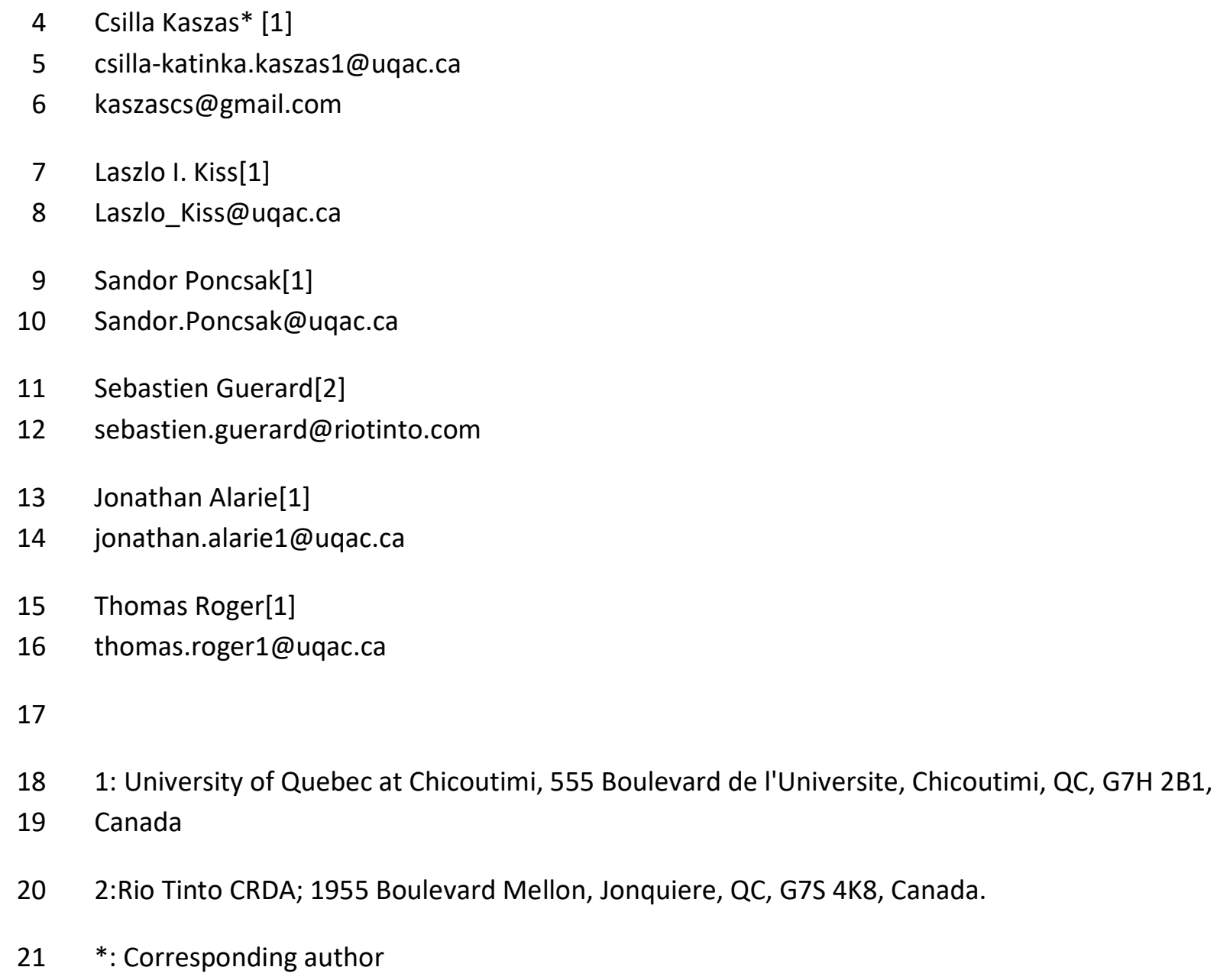

\section{Abstract}

23 A model of flotation was developed and applied to alumina in the aluminum electrolysis cell.

24 The conditions of flotation for alumina on the cryolitic bath surface and bath-metal interface 25 were determined for disc and sphere geometries. The contact angle between alumina and 26 cryolitic bath, which had not been found in the literature, was measured and it was found to be 27 around $30^{\circ}$. Experiments with compressed alumina discs on the cryolitic bath surface were 28 conducted, and the results were compared to the model. The experiments showed how 29 impurities on the bath surface influence the flotation; and how a slight asymmetry in the system 30 may accelerate sinking. 


\section{Introduction}

32 To produce aluminum, alumina powder $\left(\mathrm{Al}_{2} \mathrm{O}_{3}\right)$ needs to be dissolved in molten cryolitic bath.

33 Certain behaviors of smelter grade alumina are well known in the industry, such as its tendency

34 to stay afloat on the surface of the electrolyte after the injection, as well as its potential to form

35 aggregates - frequently called "rafts". Alumina aggregates can also sink below the liquid

36 aluminum at the bottom of the cell, forming deposits (sludge). These phenomena are noted by

37 several researchers and they form part of the general knowledge of the industry, although

38 several important details have not been analyzed in depth. As the conditions for dissolution are

39 considerably different inside the agitated bath than on its surface, the problem of raft flotation

40 deserves more attention. The sludge formation below the metal pad attracted more interest,

41 because the presence of alumina on the carbon cathode influences the distribution of the

42 current and may cause operational problems. The aim of this work is to investigate the

43 conditions of flotation of alumina on the cryolitic bath surface and on bath-metal interface, and

44 to estimate the flotation limit in static conditions.

\section{Flotation}

47 According to Newton's first law, an object at rest will stay at rest as long as the sum of all forces 48 acting upon it is zero. It is known since Archimedes that a floating object is subjected to a 49 buoyant force, equivalent to the weight of the displaced fluid. The equilibrium of the 50 gravitational and the buoyant forces are sufficient to determine the floatability and immersion 51 of a large body, such as a ship, but the surface tension plays an important role in the flotation of 52 small objects (Figure 1).

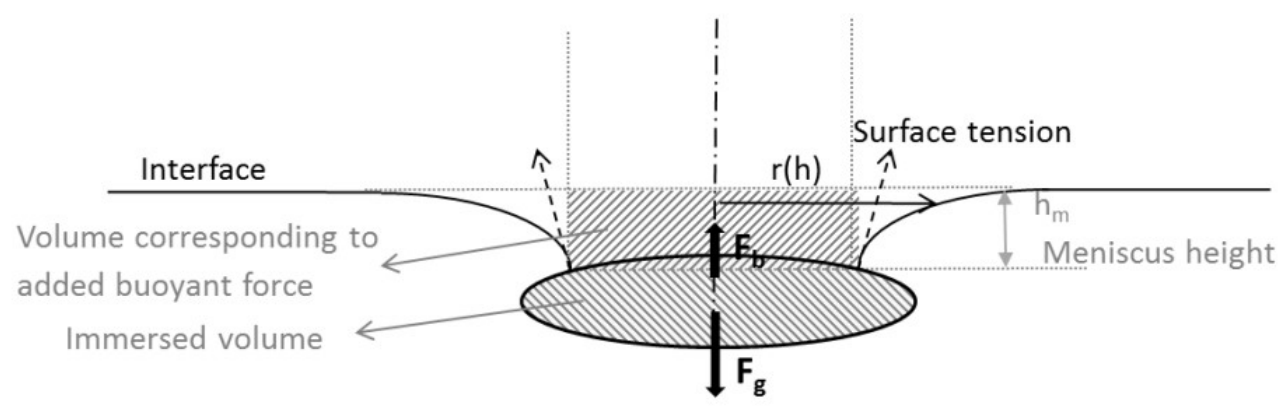

53

Figure 1: Forces acting on a floating axisymmetric object 
57 The surface tension may contribute to the flotation in two ways. The vertical component of the 58 surface tension acting along the triple line is influenced by the so-called contact angle of the 59 liquid surface at the triple line. The surface tension can aid the flotation but it can hinder it as 60 well, depending on the wetting characteristics of the liquid-solid system. The meniscus around a 61 floating object is also the result of the surface tension and it influences the hydrostatic pressure, 62 therefore the buoyant force acting on the object.

\section{Meniscus height}

64 The surface tension is the result of the cohesive forces between the molecules of a liquid. Molecules on the surface lack neighbors on one side, so the cohesive forces act on the liquid side only and pull the surface towards the bulk of the liquid. It leads to a curved surface, also called meniscus. The connection between the curvature of the meniscus and the pressure

$$
\Delta p=\gamma\left(\frac{1}{R_{x}}+\frac{1}{R_{y}}\right)=\gamma 2 K
$$

70

71

72

73

74
$\Delta \mathrm{p}$ : Laplace pressure

$\gamma$ : surface tension

$R_{x}, R_{y}$ radius of curvature in two perpendicular axes in the tangent plane to the surface.

$\mathrm{K}$ : mean surface curvature

The inclusion of the meniscus height in a flotation model can be done in various ways. The simplest solution is to neglect the meniscus height altogether, and consider the buoyant force corresponding to the volume of the object below the triple line [1] or below the undisturbed surface [2]. It is a pragmatic solution, which results in a negligible inaccuracy for the flotation of particles significantly smaller than the capillary length (Eq. (7)) of the interface, as is the case with the flotation of individual smelter grade alumina particles on the surface of the electrolyte. Solheim applied the meniscus height calculated beside a plane wall to determine the flotation limit of disc-shaped alumina agglomerates on the bath-metal interface [3]. The meniscus height around a cylinder approaches that around a plane wall as the radius increases, which makes this estimation appropriate for large objects. The most precise method would be to solve the YoungLaplace equation. Singh used direct numerical simulation of the water-air interface to determine the behavior of floating particles, and as he pointed out, the required computational capacity increases unfeasibly when there are several objects on the surface [4].

In this work the equation describing liquid surface around axisymmetric objects [5] was solved numerically to obtain the meniscus height Eq. (2):

$$
K=\frac{r^{\prime \prime}}{\left(1+r^{\prime 2}\right)^{3 / 2}}-\frac{r^{\prime}}{(b+r)\left(1+r^{\prime 2}\right)^{1 / 2}}
$$


91 Where:

92 b: radius of axisymmetric object at the triple line

$93 \quad r=r(h)$ : interface profile, (see Figure 1)

$94 r^{\prime}, r^{\prime \prime}$ : first and second derivatives of $r(h)$

95 h: meniscus height/depth, axial (vertical) coordinate

96 From Eq. 1, the surface curvature can be expressed as a function of the capillary pressure, which

97 is proportional to the meniscus height $(-h)$. Matlab ordinary differential equation solvers can

98 only solve fist order equations; therefore the second order equation was rewritten as an

99 equivalent system of first order equations. The meniscus height was in question; an initial value

100 was specified, then trial and error method was used to obtain the final result.

$101 \quad$ Angle of interface at triple line

102 The angle of the interface at the triple line is necessary to find the vertical component of the 103 surface tension, and also as a boundary condition for the Young-Laplace equation to determine 104 the meniscus height. For smooth, homogenous surfaces, the Young-Dupré equation shows the 105 connection between the interfacial energies and the equilibrium contact angle. If the floating object has a smooth surface, the angle of the object at the triple line and the contact angle determine the angle of the liquid surface Eq. (3)

$$
\gamma_{S G}-\gamma_{S L}=\gamma_{L G} \cos \alpha
$$

The contact angle $(\alpha)$ has a significant influence on the flotation. A large contact angle will permit the floating object to descend deeper below the line of the undisturbed surface without sinking than an object wetted by the liquid could. But that is not to say flotation of an object denser than the liquid is not possible even if the contact angle defined by the Young-Dupre equation would reach $0^{\circ}$, as the shape and surface quality of the solid would also influence the angle of the interface at the contact line ( $\delta$ ) (Figure 2: Illustration for Gibbs extension to YoungDupré law.

In the mathematical model, two different geometries were considered: spheres and cylinders (round disks). The spherical objects were considered smooth, but the effect of roughness can be investigated by increasing the apparent contact angle in the model. In case of circular disks, the angle of the interface at the edge has a degree of freedom as specified by Gibbs [2] Eq. (4).

$$
\alpha_{0}<\alpha<180-\beta+\alpha_{0}
$$

Where $\alpha_{0}$ is the static equilibrium contact angle and $B$ is the angle of the corner of the solid body as shown in Figure 2. 


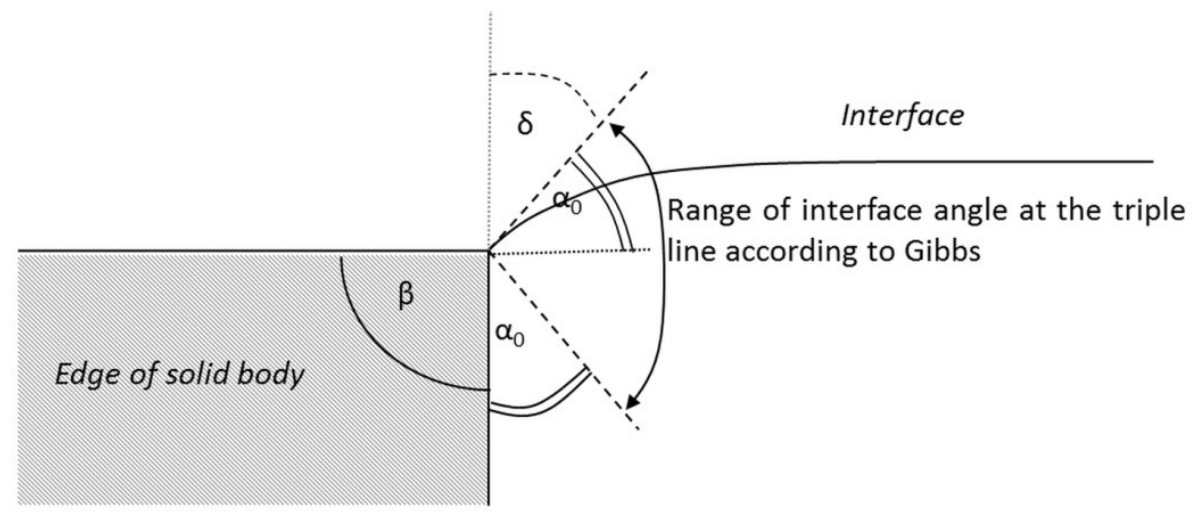

Figure 2: Illustration for Gibbs extension to Young-Dupré law

On rough or heterogeneous surfaces, the Young equation is only locally applicable, although the Wenzel and the Cassie-Baxter equations offer estimations for the apparent contact angle ( $\alpha$ ) for different wetted states. [5]

To determine the angle of the interface at the contact line, both the shape of the object and the contact angle should be known, however it is often not the case. Maru's approximation for maximal radius of a floating spherical object is independent of the contact angle. His equation has been used to calculate the flotation limit of solid alumina agglomerate on the bath-metal interface. As the result, $7.5 \mathrm{~mm}$ was calculated as the critical diameter [6].

\section{Flotation in mineral industry}

Particle flotation has been used in the mineral industry for more than a hundred years to separate valuable ores from the waste. The ores are crushed, and then mixed in with water and possibly other additives. Air is fed into the slurry and the rising bubbles collect and transport certain minerals to the surface, as particles get attached to the interface of the bubbles [7]. Most researchers in this field have a more practical than theoretical approach, focusing not on the possibility but the probability of flotation, the particle retention. Flotation models can be found, albeit sometimes crude and the bulk of the works is empiric [8] [9] [10]. Although there 142 are unarguably differences, it is worth pointing out that the anode gas forming in the electrolysis 143 cells also creates a current of bubbles in the electrolyte. This parallel might become even more 144 significant in the future with inert anode technology, as they work with saturated bath that 145 would promote the presence of undissolved alumina.

146 Flotation of alumina in the cryolitic bath, along the bath-metal interface 
Generally, the analysis of the flotation conditions on the bath surface has not generated much interest. Walker measured the density of alumina rafts and found that they can float even when their density exceeds the density of the cryolitic bath [11]. The flotation of carbon particles on the bath, and their interference with the measurement of the surface tension, noted by Kucharic [12] is worth mentioning as the same issue was encountered by the authors of this paper, and industrial cells are contaminated with carbon dust even more than experimental 153 setups.

The possibility of flotation of alumina agglomerates on the bath-metal interface is of more direct importance to the industry. A flotation model of disc-shaped agglomerates has been presented by Solheim [3], and a simple estimation for the flotation limit of spherical agglomerates by Thonstad [6]. Both of them focused mainly on the possibility of an alumina particle layer on the interface. They theorize that the alumina layer on the interface impedes mass transfer of aluminum, therefore decreases the re-oxidation of the metal by the anode gas and increases current efficiency. Solheim proved the possibility of alumina and cryolite precipitation on the interface through theoretical investigation of bath chemistry [13], though he later pointed out that Marangoni convection might clear the interface of those particles [3].

Properties of alumina-bath system, input data for flotation model

The values of density, interfacial tension and contact angle are necessary for the calculation of forces on the interface. The density of the electrolytic bath depends on its temperature and composition. To maintain good separation between the bath and liquid aluminum, the latter having a density of $2.3 \mathrm{~g} / \mathrm{cm}^{3}$, the bath density is usually kept around $2.1 \mathrm{~g} / \mathrm{cm}^{3}$ or below. Several empirical equations are available and have been presented in well-known reference books in the industry [14] [15] Eq. (5).

$$
\begin{aligned}
\rho_{\text {bath }}\left[\frac{g}{\mathrm{~cm}^{3}}\right]= & 2.64-0.0008\left(\text { temperature in }{ }^{\circ} \mathrm{C}\right)+0.18\left(\frac{w \% N a F}{w^{\circ} \% A l F_{3}}\right) \\
& -0.008\left(w \% \mathrm{Al}_{2} \mathrm{O}_{3}\right)+0.005\left(w \% \mathrm{CaF}_{2}\right)+0.008\left(w_{0 M F_{2}}\right) \\
& -0.004(w \% L i F)
\end{aligned}
$$

171 The above-mentioned values represent well the parameters of the experimental setup used in 172 the present work and shall be used in the calculations.

173 The surface tension of cryolitic melts has been measured by several research groups by pin 174 detachment method [16] or maximum bubble pressure methods [12]. Model equations were 175 fitted to the experimental data Eq.(6). The validity of these empirical equations is limited to a 176 certain temperature range where the experiments were conducted. As the alumina fed into the 177 bath tends to be several hundred degrees cooler than the bath itself, surface tension values at 178 low temperature hold particular interest in the flotation of alumina rafts. Beside measurements, 
a theoretical approach to predict the surface tension of molten salt mixtures is also available 180 [17].

$$
\begin{aligned}
\gamma_{\text {bath }}\left[\frac{m N}{m}\right]= & 266.69-0.1257 t-4.754\left(w \% A l F_{3}\right)+1.546 \cdot 10^{-3} w \% A l F_{3} \cdot t \\
& +3.002 \cdot 10^{-2} \cdot w \% A l F_{3}{ }^{2}+2.078 \cdot 10^{-5} \cdot w \% A l F_{3} \cdot w \% C^{2} F_{2} \cdot t \\
& +2.702 \cdot 10^{-5} \cdot w \% A l F_{3} \cdot w \% l_{2} O_{3} \cdot t-3.507 \cdot 10^{-4} \cdot w \% C_{2} F_{2} \\
& \cdot w \% \mathrm{Al}_{2} O_{3} \cdot t
\end{aligned}
$$

where $t$ is the temperature in Celsius.

The equation (Eq. 6) predicts the surface tension to be $145 \mathrm{mN} / \mathrm{m}$, at the chemical composition and the temperature of the bath, during our experiments [16]. For the bath-metal interfacial tension, $450 \mathrm{mN} / \mathrm{m}$ was used in the calculations [18].

For future reference, the capillary length (a), a scaling factor necessary to calculate surface curvature, and interpreting dimensionless forms of the flotation model, is $2.6 \mathrm{~mm}$ at the upper bath surface Eq. (7).

$$
a=\sqrt{\frac{\gamma}{\Delta \rho g}}
$$

The contact angle between alumina and aluminum on the BMI was measured by Utigard [20] and was found to be between $150-170^{\circ}$. As the electrolytic bath dissolves alumina, it is expected that alumina is wetted by the bath. However, the actual contact angle between these substances has not been found in the literature.

\section{Experimental}

196 Setup

197 The high temperature experiments were conducted at the laboratory of the authors' research 198 group, GRIPS, UQAC, in a carbon crucible. The free surface of the bath was $7.6 \mathrm{~cm} \times 7.6 \mathrm{~cm}$ square with rounded corners. The depth of the bath was $3.8 \mathrm{~cm}$. The prepared bath contained $83 \%$ cryolite, $11.5 \% \mathrm{AlF}_{3}$ and $5.5 \% \mathrm{CaF}_{2}$. Due to impurities, the initial alumina content was $3 \%$. The rectangular carbon crucible was placed in the heated chamber of an electric kiln, and

203 heated from three sides. The thermocouple for the temperature control was placed under the crucible. The insulated cover from the top was removed during experiments, and replaced between tests so the temperature could stabilize. For longer experiments, to reduce the heat loss, the opening was covered with a quartz plate between manipulations to be able to observe the test object on the bath surface. The control temperature usually was set to $980{ }^{\circ} \mathrm{C}$, which 
decreased after the removal of the cover. Periodically a thermocouple was inserted in the bath and it showed that the temperature stabilized $10^{\circ} \mathrm{C}$ below the control temperature.

\section{Contact angle measurement}

211 The contact angle is a necessary parameter to determine the conditions of flotation. As the 212 alumina-cryolitic bath contact angle has not been found in literature, it was necessary to obtain 213 it through experiments. The high temperature and corrosive nature of the bath renders visual 214 methods to determine contact angle nearly impossible. Another difficulty may rise from the low 215 superheat: bath would solidify in contact with insufficiently preheated alumina. Extended 216 contact between unsaturated bath and alumina leads to dissolution, changing the shape and 217 size of the alumina object.

218 The first attempts to measure the contact angle by the capillary rise method provided 219 inaccurate results. To improve precision, other tests with Wilhelmy method were prepared, 220 using the gravimetric system developed by GRIPS at UQAC [19].

221 The gravimetric system was developed to analyze different aspects of the behavior of alumina in 222 the cryolitic bath (Figure 3). Different test objects were suspended above the cryolitic bath. The 223 samples were descended at a predetermined velocity into the bath while their apparent mass 224 was measured by a load cell. Both the position and the apparent mass were recorded 225 simultaneously.

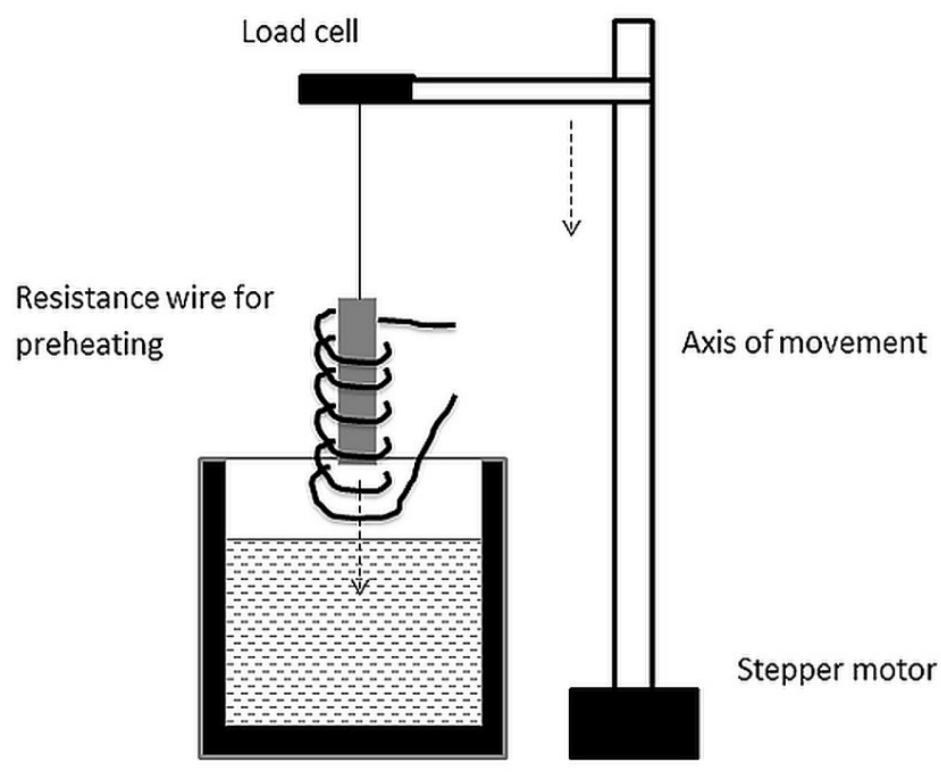


229 An alumina rod of $1 / 4$ inch diameter was suspended by nichrome wire above the bath surface, preheated to avoid bath solidification upon insertion, then descended with a velocity of 0.5 $\mathrm{mm} / \mathrm{s}$, the position and resultant force logged at 1 second intervals.

232 Before the test object touched the liquid surface (position larger than $\mathrm{p}_{0}$ ), the force corresponds to its mass. When the object reaches the surface, the resultant force increases rapidly $(\Delta \mathrm{F})$, due to the effect of the surface tension. With further descent in the liquid (position below $p_{0}$ ) the resultant force is decreased by the buoyant force.

$$
\begin{gathered}
F=m \cdot g+\left\{\begin{array}{c}
0 \text { if } p=\text { position }>p_{0} \\
d \pi \gamma \cdot \cos \alpha+\frac{d^{2} \pi}{4} \rho_{l} \cdot\left(p-p_{0}\right) g \text { if } p \leq p_{0}
\end{array}\right. \\
\cos \alpha=\frac{\left.\Delta F\right|_{p_{0}}}{d \cdot \pi \cdot \gamma}
\end{gathered}
$$

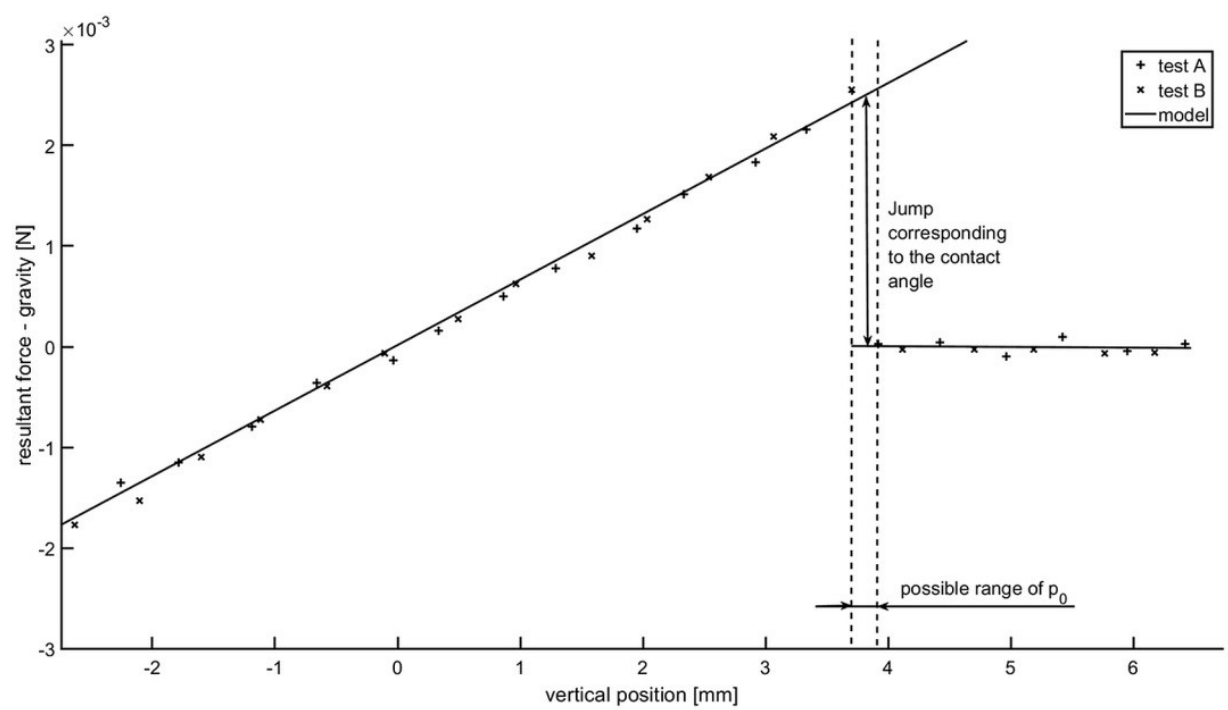

Theoretically, the contact angle can be calculated from the jump in the resultant force when the rod first touches the surface ( $p_{0}$, position 0$)$. However, as the data is not continuous, the detection of the jump may be delayed by almost a full-time step. To decrease this uncertainty, the results of two tests ( $A$ and $B$ ) were overlapped (Figure 4). Note that as we cannot produce perfectly identical samples, both the resultant force and the zero position need to be adjusted.

Figure 4: Buoyant and surface tension forces acting on an alumina rod, immersed into cryolitic bath Vertically, one set of results was shifted with the force corresponding with the difference between the initial masses of the samples, while the horizontal shifting aligned the left side of 
the diagram. This process did not only provide better visual representation, but the exact position of zero was narrowed down significantly. There is a slight noise in the data, but the test results correspond well to the model, and it narrows down the range of the contact angle to a range of $29-34^{\circ}$.

Primary alumina discs (with 40mm diameter and $8.6 \mathrm{~mm}$ thickness) were prepared using a Struers Labo-Press 3 machine. $12.5 \mathrm{ml}$ powder was fed into the machine chamber, and exposed to $50 \mathrm{kN}$ for 15 minutes which included a 3 minutes initial period where the powder was heated to $180^{\circ} \mathrm{C}$. The produced discs were very fragile, they crumbled easily and some powder got lost every time during handling them. For this reason, a separate batch of discs was prepared only for the statistical analysis of the properties of the discs. The average mass of a dozen discs was $13.4 \pm 0.2 \mathrm{~g}$. The average mass loss after four manipulations (for example: taking it up and putting it on a scale would count as one manipulation) was $1.2 \%$. The average thickness of the discs is $8.6 \pm 0.1 \mathrm{~mm}$. The density derived from these measurements was $1.25 \mathrm{~g} / \mathrm{cm}^{3}-$ while the measured (tapped) bulk density of alumina powder used for the preparation of discs was 1.03 $\mathrm{g} / \mathrm{cm}^{3}$, calculated by measuring the mass of a given volume of powder. Three successful series of tests, noted 'A', 'B'and 'C' were each conducted consecutively with fresh cryolitic bath.

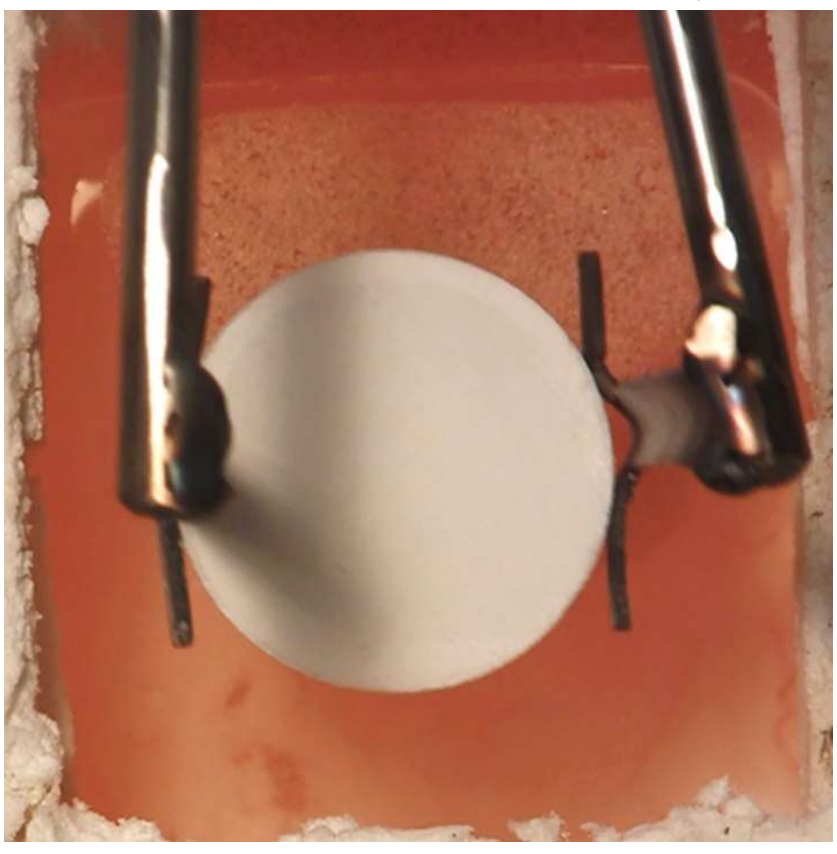

The compressed discs were placed on the surface of cryolitic bath (Figure 5), and after a certain period of time, they were recovered. The apparent density of the samples was estimated by 
272 form, while in some cases, samples were covered with a silicon-based spray to obtain density 273 with Archimedes method; by weighing of the impermeable sample suspended in water and in 274 air.

275 Experiments on the bath-metal interface were attempted, but visual observation failed due to a 276 rapidly developing aluminum fog in the cryolite following the addition of the metal. The 277 formerly transparent cryolitic bath turned opaque, and the samples were hid from view under 278 the bath surface. While the gravimetric method may be applied in the future to examine the flotation on the BMI, it is recommended to apply measures that help flatten the bath-metal interface.

\section{Mathematical model}

282 The mathematical model was developed for two shapes: spheres and circular disks. The former 283 is used as a universal shape; it can represent a single alumina particle, or an agglomerate on the 284 bath-metal interface. The disk form holds more interest with small height-diameter ratio, as 285 alumina tends to spread on the bath surface, therefore a disc is a reasonably good 286 representation of an alumina raft. Theoretically, the same equation can be applied for large 287 height-diameter ratio, such as a rod, floating vertically. This, admittedly, is an unlikely, mostly unstable scenario to occur naturally, and since it has no relevance for the studied phenomena either, the height-diameter ratio in this study was limited to 1 and under.

The governing equations of flotation are presented below, along with the corresponding shapes

(Figure 6).

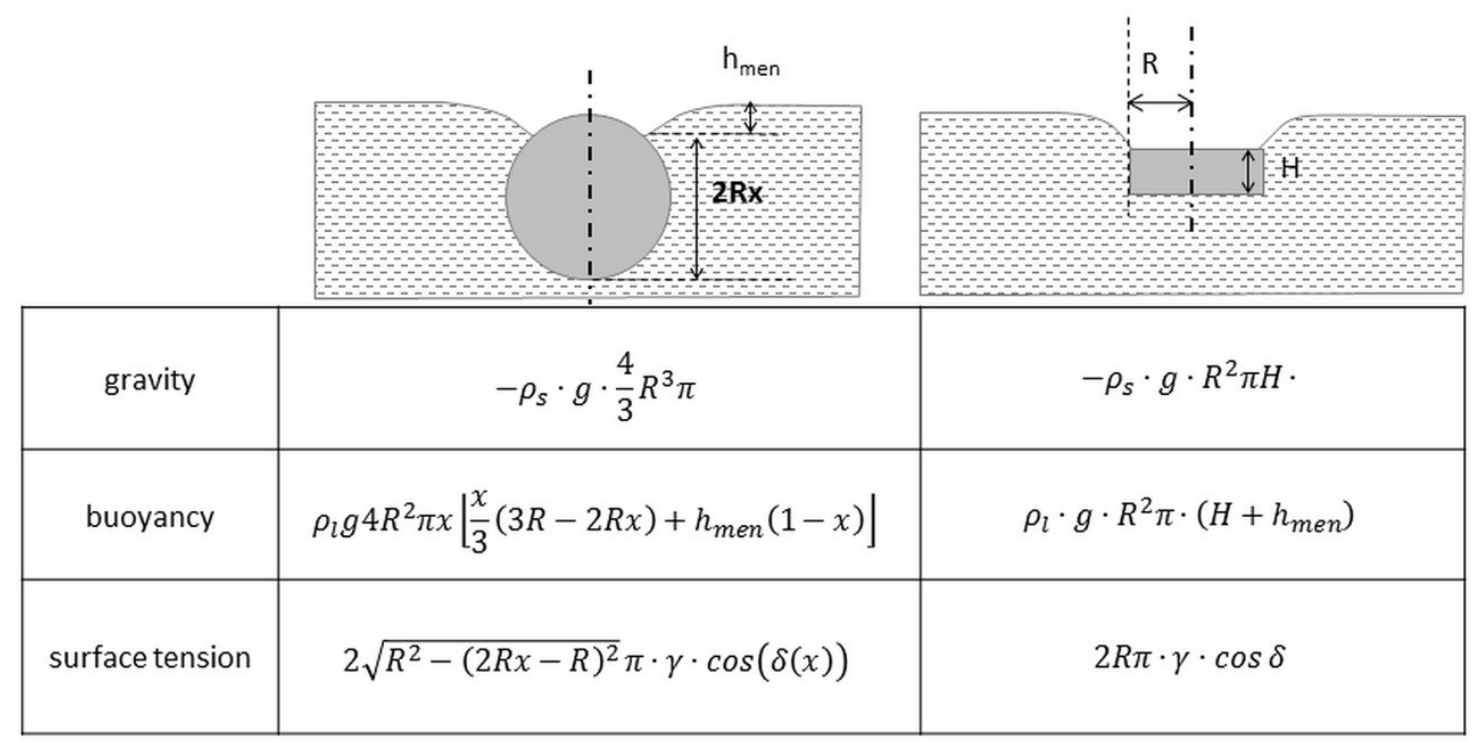

Figure 6: Geometries and corresponding force terms used in the present flotation model 
Where $\rho_{s}$ is the density of solid,

$296 \rho_{1}$ is the density of liquid,

$297 \quad \mathrm{R}$ is the radius of the sphere or the disc, respectively

$298 \mathrm{H}$ is the thickness of the disc

$299 \mathrm{x}$ is the relative immersion of the sphere: the vertical distance between the triple line and the

300 bottom of the sphere, in relation to its diameter

301 and $\mathrm{h}_{\text {men }}$ is the meniscus height.

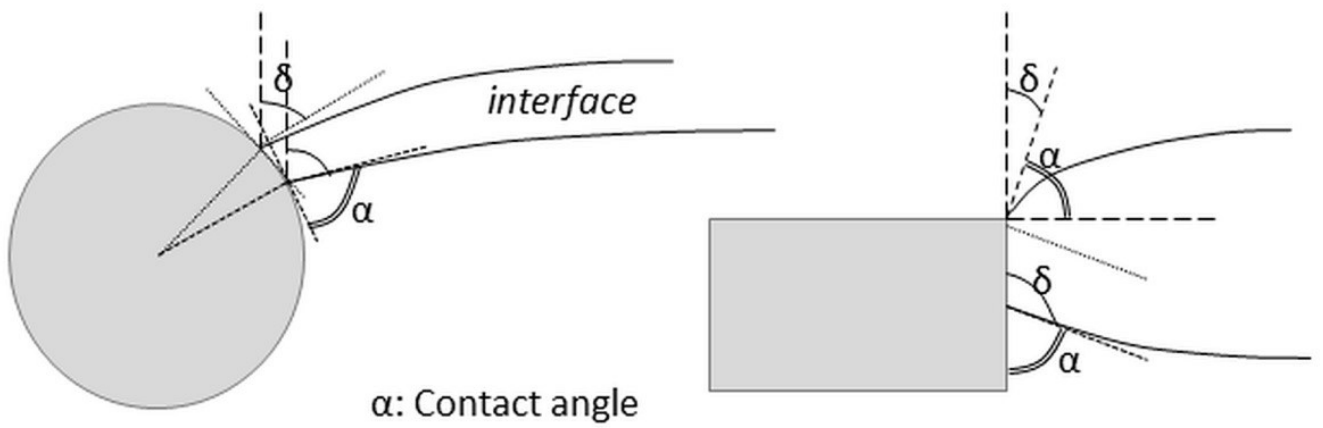

$\delta$ : Angle of interface at the triple line
307

308

309

310

311

312

Figure 7: Dependence of the angle of interface on the relative immersion with different geometries

Based on the relative immersion of a sphere, $x$ and the apparent contact angle $\alpha$, the angle of the interface at the triple line can be expressed by the following equation.

$$
\delta(x)=2 \pi-\arccos (1-2 x)-\frac{\pi}{2}-\alpha
$$

314 With disc geometry, the flotation limit shall be reached at total immersion, where the angle of 315 the interface has a degree of freedom described by the earlier mentioned Gibbs extension to 316 the Young-Dupre law (Figure 2). The meniscus height was determined by the numerical solution 
317 of the formerly presented equation (2). The following boundary conditions should apply, 318 assuming a single floating object on an otherwise undisturbed surface:

$$
\begin{aligned}
\lim _{h \rightarrow 0} r(h) & =\infty \\
\left.\frac{d r}{d h}\right|_{h=-h_{m e n}} & =\tan \delta
\end{aligned}
$$

320 Due to the limitations of Matlab, and the fact that $h_{\text {men }}$ was the sought parameter, a trial and 321 error method was applied, and the meniscus height gradually decreased until the solution 322 fulfilled (or rather, approached) the first boundary condition. Solutions for a few different 323 variables are presented below (Figure 8).

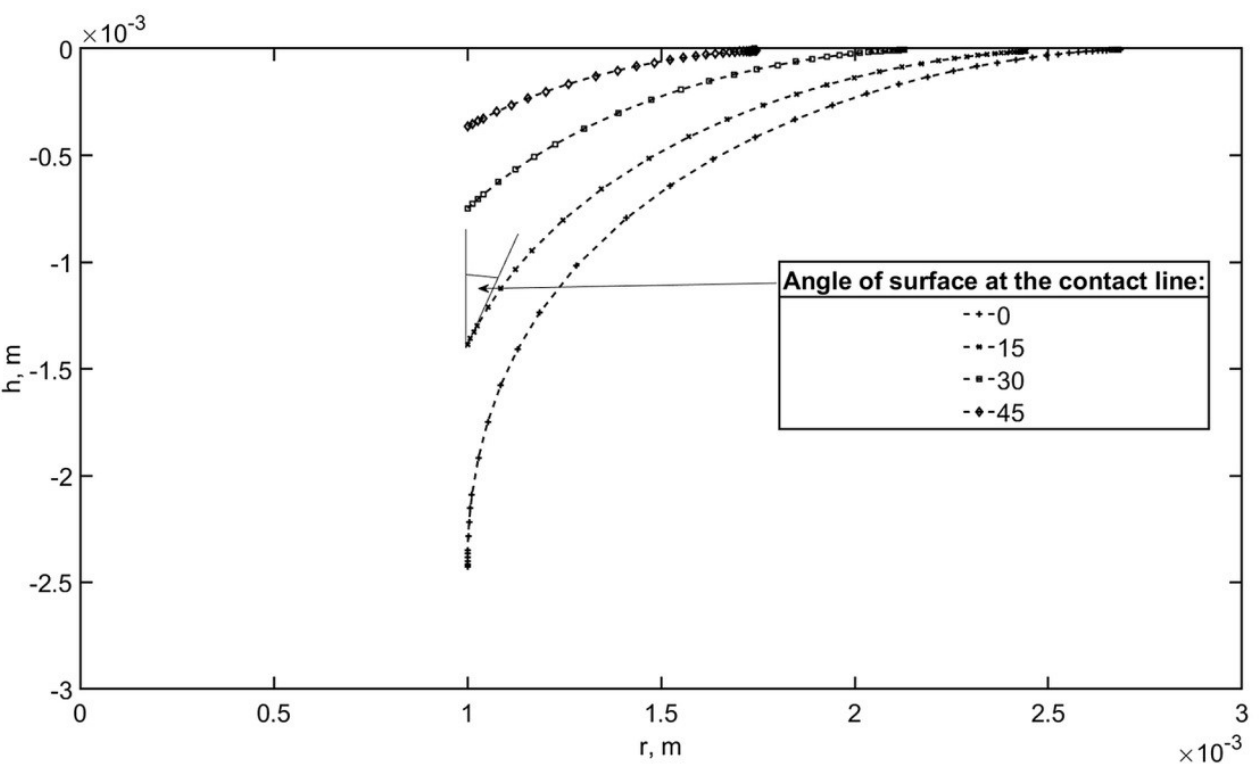

Figure 8: Numerical solution of differential equation, shape of meniscus

328 negative, so as the interface would bulge over the floating object. This does not hinder the 329 solution of the differential equation Eq. (2) (i.e. finding the shape of the interface) for larger 330 diameters, but as the radius of the triple line fell below the capillary length, the range of angles 331 for which acceptable solutions were found, became limited (Figure 9). 


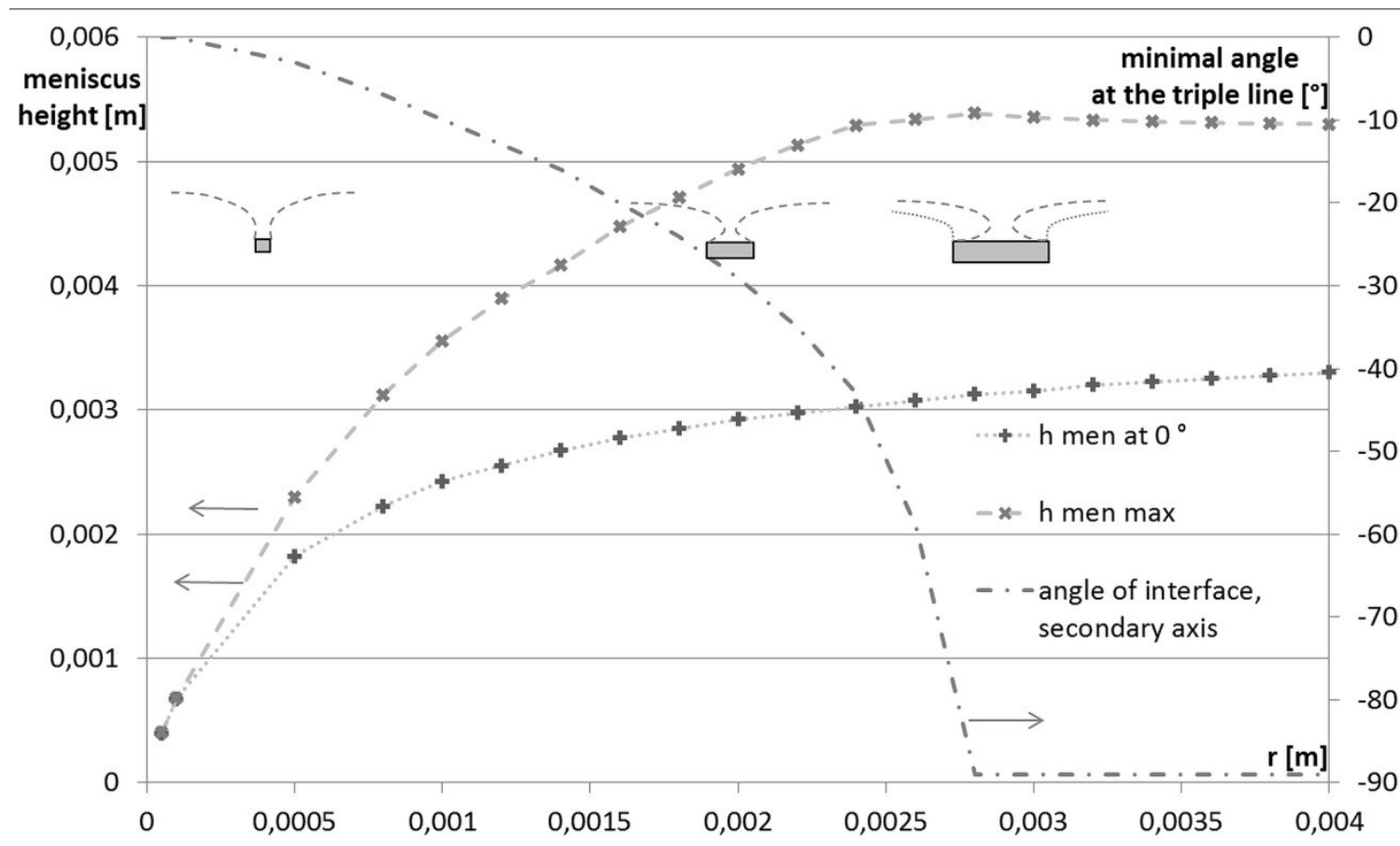

Figure 9: Dependence of meniscus height on radius and angle of surface

334 It should be noted however, that researchers who attempted solving the problem of meniscus shape, did not succeed achieving negative interface angles with floating discs in reality, supposedly due to instability [2]. It stands to reason, that a position with negative surface angle would be unstable, and a slight tilt of the floating disc could swing the disc out of balance. As one side of the disc gets closer to the surface, the angle of surface of the contact line at that side gets closer to vertical, escalating the tilt. For this reason, the minimal angle of the surface for discs in the model was limited to 0 degree (Figure 10).

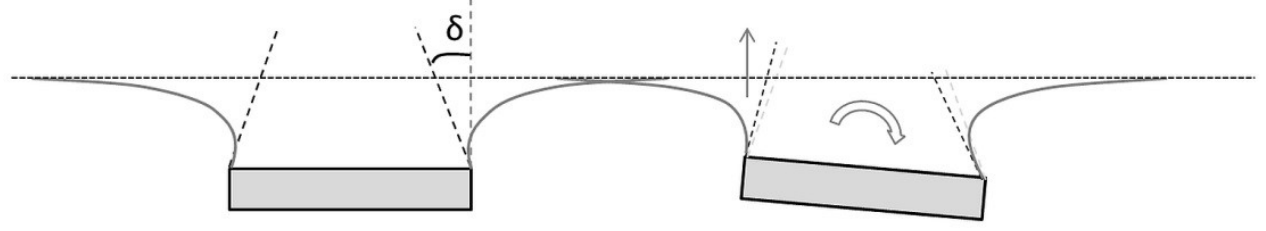

Figure 10: Unstable position of a floating disc

343 While there is no possibility of stable floating position when the density of the solid exceeds the 344 density of the liquid, metastable states exist, where depending on the magnitude of the 345 disturbance, the degree of tilting or displacement from the equilibrium position, the floating 346 object might return to its original floating position, or sink. 


\section{Interpretation of results}

348 Firstly, the model results for flotation limit are presented in a general, dimensionless form 349 (Figure 11). The contact angle has an immense effect on the flotation limit of spheres. While in 350 case of perfect wetting, the density of a floating sphere could not exceed the density of the 351 liquid, the maximal density gradually increases with increasing contact angle, with a more rapid 352 rate in case of smaller dimensions (when the radius of the spherical object is at the same scale 353 as the capillary length).

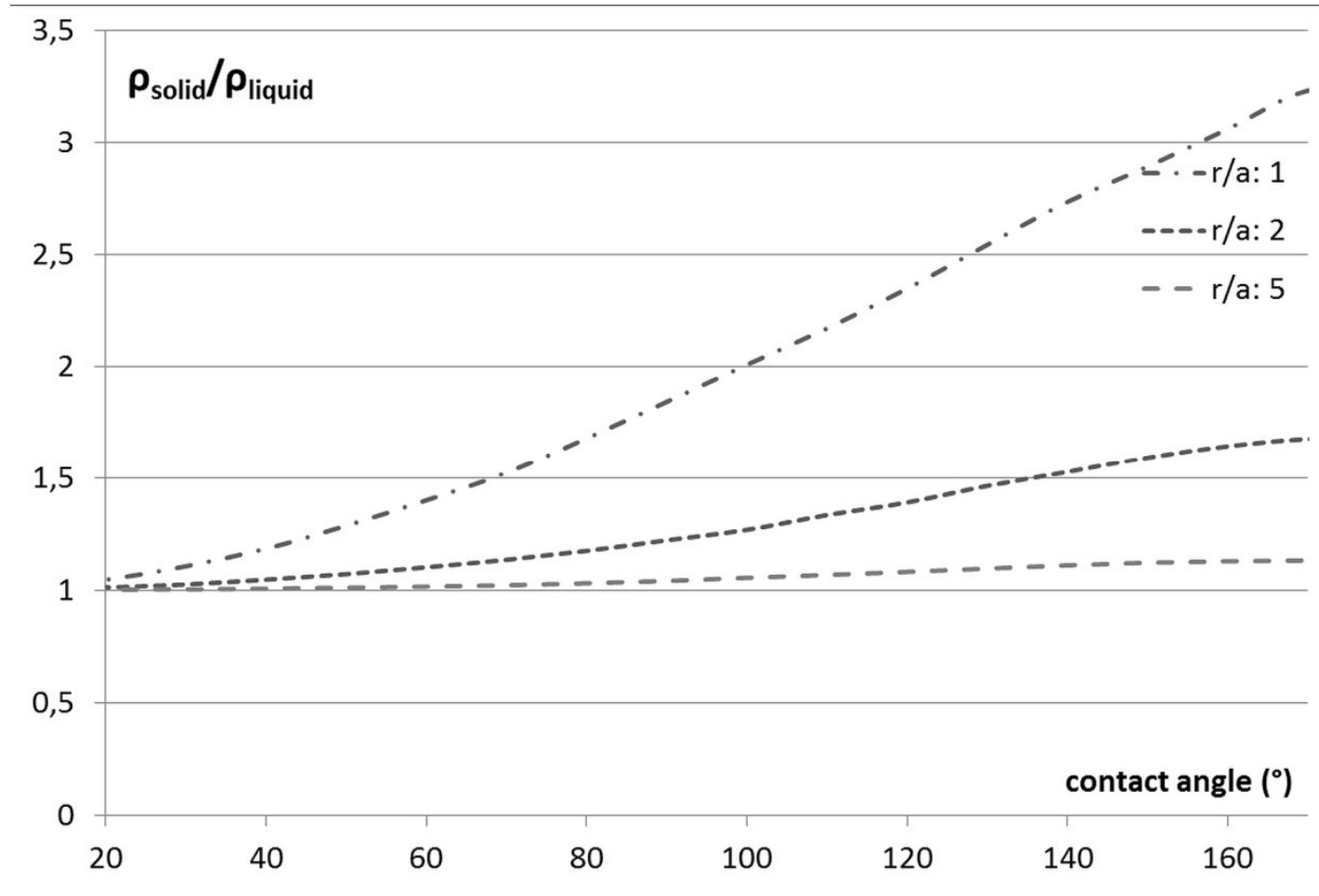

Figure 11: Flotation limit of spheres

357 For studying the behavior of solid disks and spheres on the upper surface of the bath, the 358 flotation limit was calculated for $30^{\circ}$ contact angle. The effect of the small contact angle is more 359 prominent for the flotation limit of spheres than for discs. Discs would always have a higher 360 flotation limit than spheres with the same volume and diameter; and as the height-diameter 361 ratio decreases, the discs get thinner, the curved surface's contribution to the buoyant force 362 becomes more prominent, as can be seen in the increase of flotation limit with decreasing $\mathrm{h} / \mathrm{d}$ 363 ratio (Figure 12). 


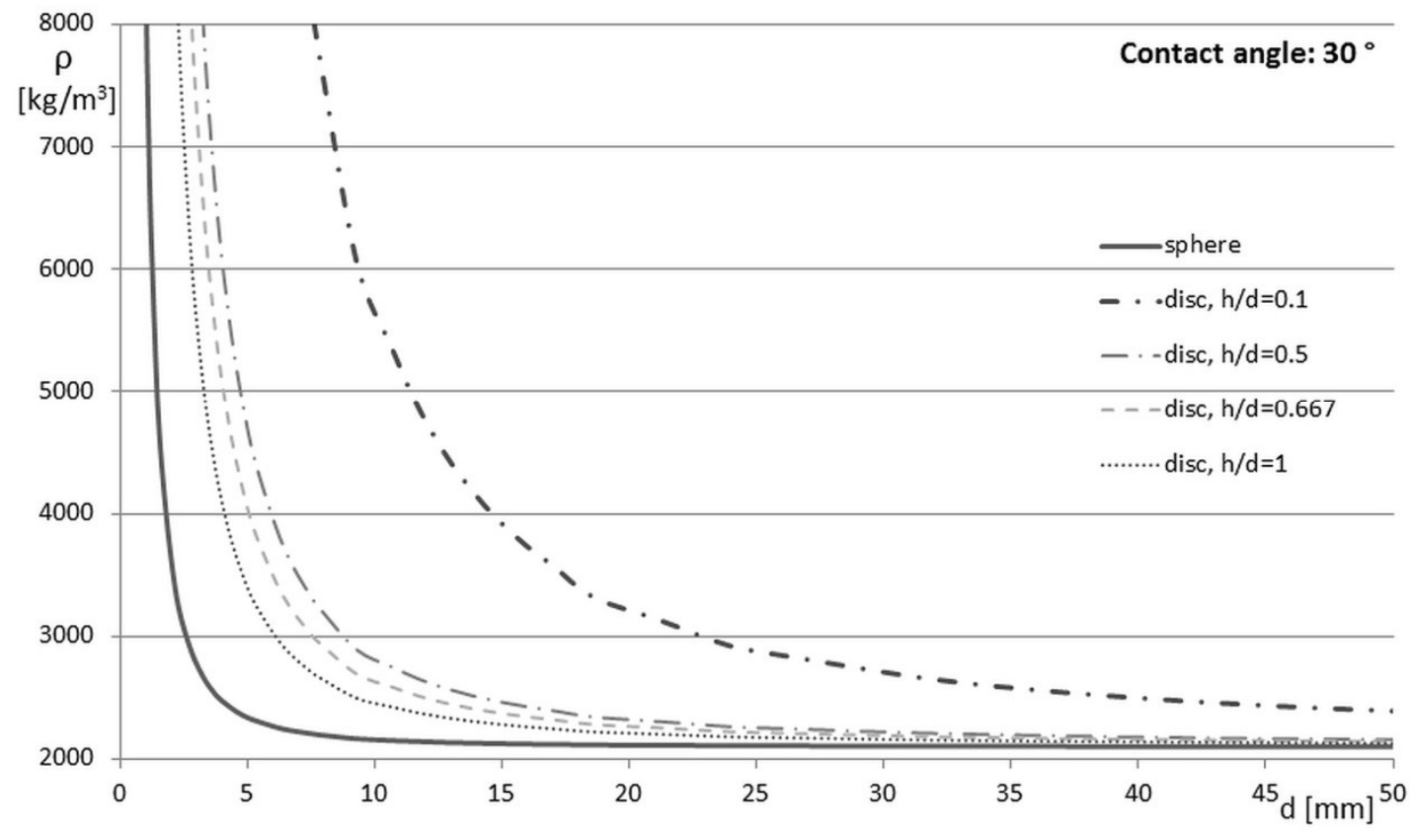

Figure 12: Flotation limit on bath surface, contact angle: $30^{\circ}$

Alumina powder tends to spread on the bath surface due to its moisture content that evaporates when it gets in contact with the molten electrolyte. Therefore, discs with small thickness-diameter ratios hold the most interest. The bulk density of alumina powder is around $1 \mathrm{~g} / \mathrm{cm}^{3}$, while the skeletal density of alpha alumina is almost $4 \mathrm{~g} / \mathrm{cm}^{3}$. While cylindrical 370 agglomerates with large height-diameter ratio could be expected to float if they are only a few $371 \mathrm{~mm}$ in diameter, thin rafts could theoretically stay afloat easily. 


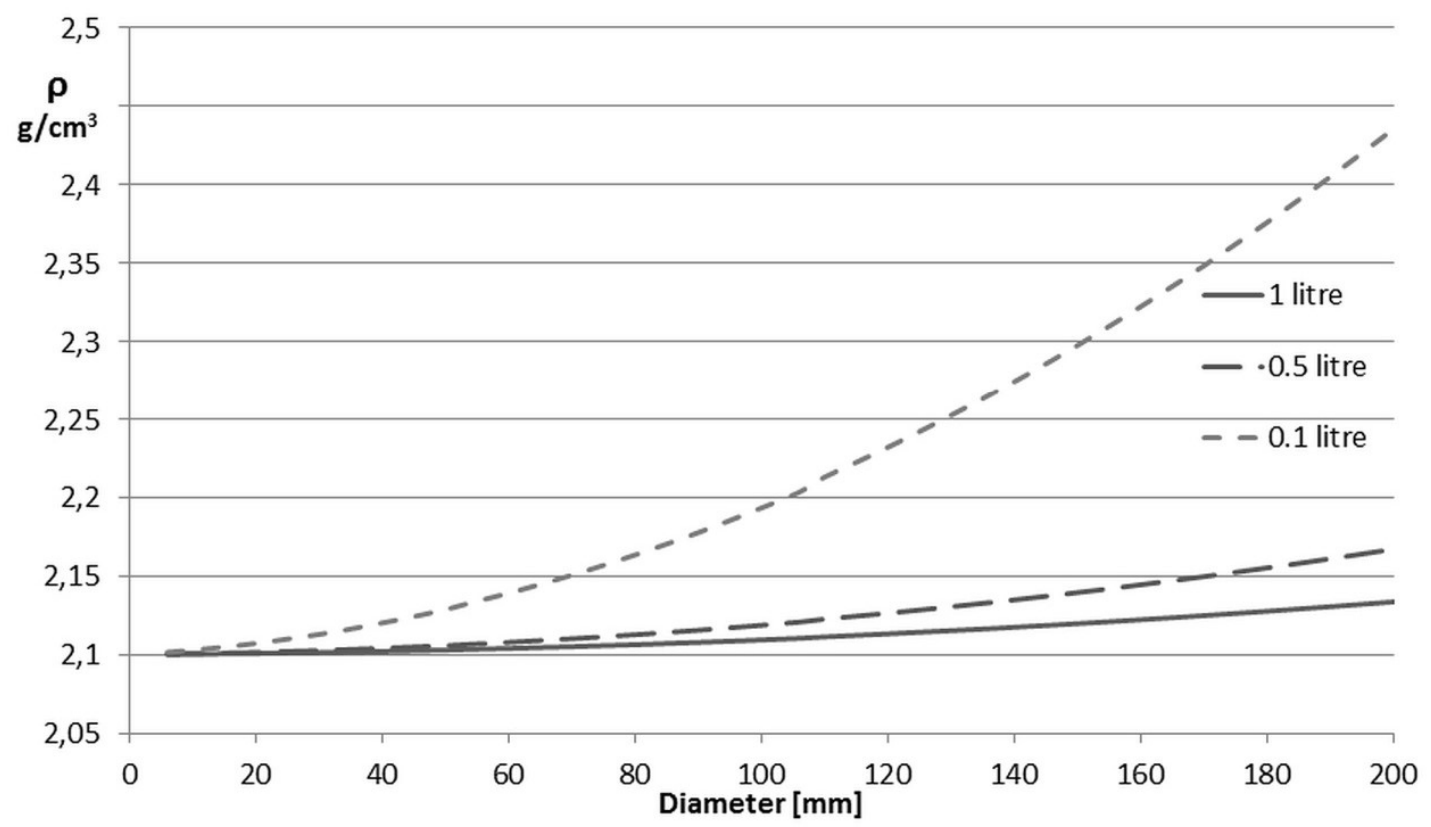

Figure 13: Density limit of a fixed volume of alumina raft, assuming spreading to disc-shape In industrial electrolysis cells a certain quantity of alumina powder is injected to the bath surface at a time, usually between 0.5 and $2 \mathrm{~kg}$, which corresponds to $0.5-2 \mathrm{I}$ of volume. This powder tends to spread on the available surface, limited by the anodes. With these considerations, the density limit of flotation was calculated for disc-shaped alumina rafts (Figure 13). While the density of larger rafts could only slightly exceed the density of the bath before sinking, with smaller batch-sizes, the maximal density increases rapidly with the available surface for spreading.

\section{Comparison of model and experiments}

The experiments proved that alumina discs can float on the surface of the cryolitic bath, even when their density became larger than the density of the bath, therefore the surface tension can play a role in the delayed dispersion of alumina in the bath. In the evaluation of the experiments, the mathematical model of flotation is used reversibly. When modeling the flotation, the maximal density of a floating object was calculated, based on its size and the contact angle. For the experiments, the density and dimensions of the floating discs are known, which, with the help of the mathematical model, provides the angle of the surface at the contact line, at the moment the disc was removed from the bath - and that points to the minimal contact angle (Figure 14). The discrepancy between the estimated and measured density are due to the fact that the discs were covered with an uneven layer of frozen bath, 
partly due to the removal process. As the estimated volume was based on the diameter and thickness of the discs at their bottom corners, the volume was underestimated. While for the measurement of the volume with Archimedes method, the total volume was measured, including the added frozen bath, which adds to the volume, and, at that point of infiltration, decreases the overall density.

While the contact angle between alumina and bath was measured to be around $30^{\circ}$ using the Wilhelmy method with an alumina rod, with the discs, only $10^{\circ}$ was reached at best. This could be explained by several factors relating to the realities of experimentation, such as the lack of a well-defined, sharp edge of the discs, slight asymmetries in either the alumina discs or the setup, and the continued exposure to disturbances, the convection in the bath, and manipulations around the setup.

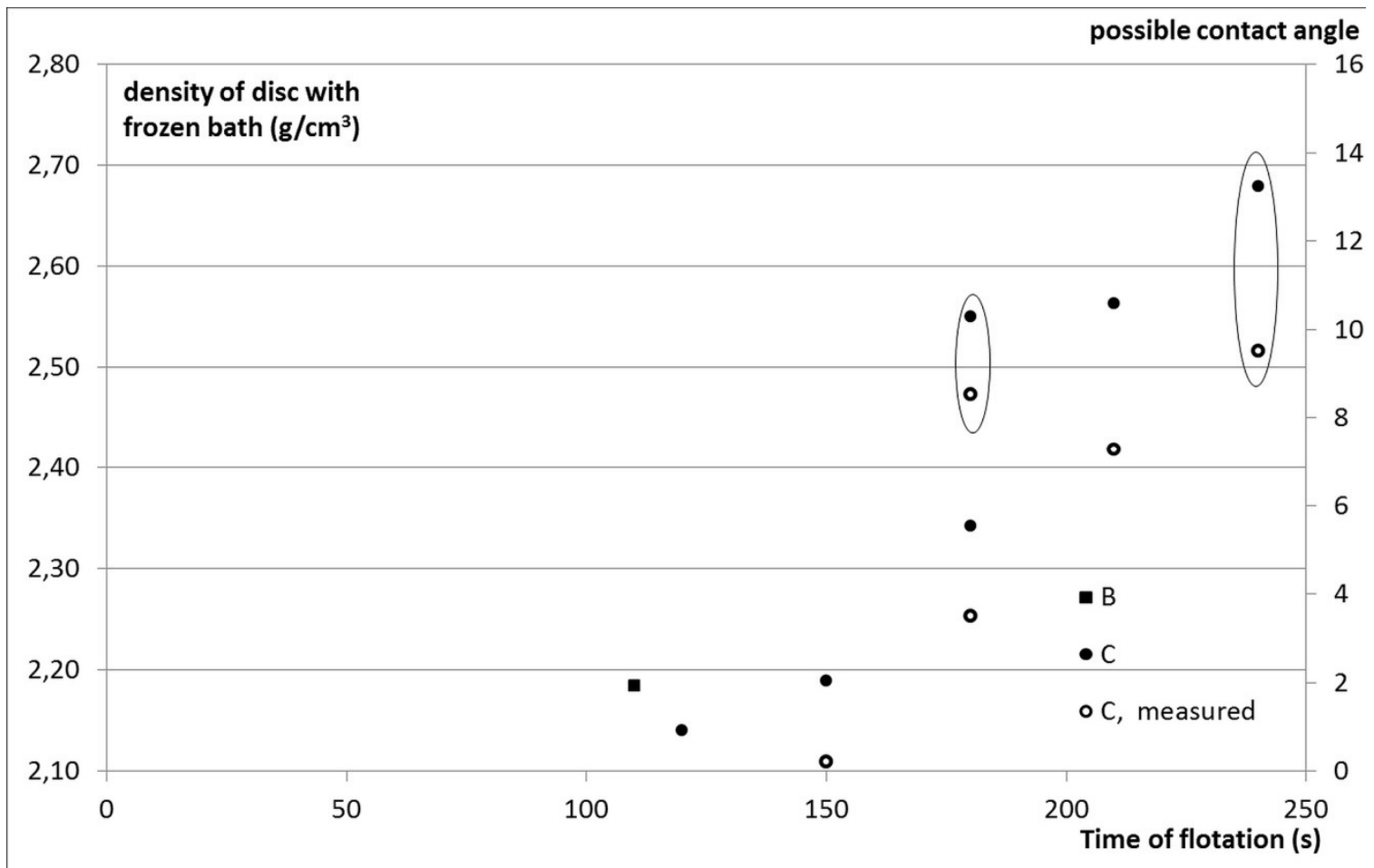

Figure 14: Density of samples, results from repeated experiments B and C

407 The video recordings of the experiments also show an influential factor, unaccounted for in the 408 mathematical model: the presence of carbon and other particles on the bath surface, originating 409 from the degradation of the carbon crucible and from the refractory walls. As the selected series 410 of images show (Figure 15), the surface starts creeping over the disc at the side that is clear of 411 scum. While the impurities on the left side hold the liquid surface at bay, the latter creeps upon 412 the disc on the right with an accelerating rate, causing its eventual submersion. 

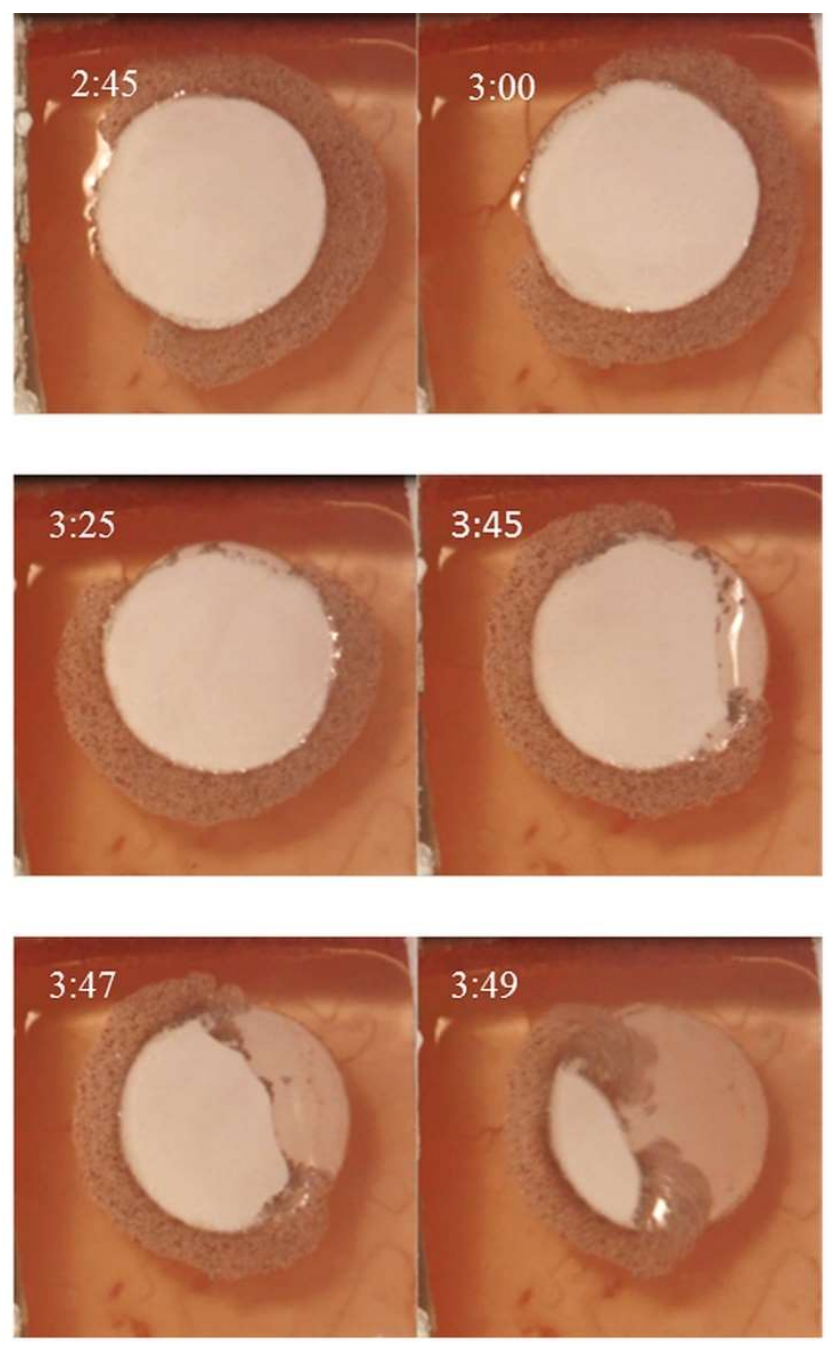

Figure 15: Compressed alumina disc, process of sinking; time after insertion marked (mm:ss)

\section{Conclusions}

419 The contact angle between alumina and cryolitic bath was measured, and a range of $29-34^{\circ}$ was

420 determined.

421 A mathematical model was developed to investigate the conditions of static flotation of

422 axisymmetric objects on a liquid surface. Two geometry, disc and sphere, were considered. The 423 model was specifically applied to the parameters encountered in an aluminum electrolysis cell, 424 to analyze the flotation limit of alumina rafts, forming on the bath surface upon the injection of 425 alumina powder. The flotation limit was defined as the maximum density for a floating object of 426 a given size; alternatively, the flotation limit could be formulated as the maximum size of a 427 floating solid for a given density. It was shown how the expected shape, size and density of 
428 alumina rafts and agglomerates could influence their flotation. Realistic sizes of alumina batches

429 were considered and the effect of spreading on the flotation was presented.

430 Besides a mathematical model, experiments with artificial alumina rafts were conducted on the

431 surface of cryolitic bath. While the theoretical flotation limit for compressed alumina discs has

432 not been reached in the experiments, it was only expected since perfect edges and symmetry is

433 unobtainable in reality, nevertheless, the contribution of the surface tension to the flotation was

434 shown.

435 The experiments brought attention to the influence of solid particles on the bath surface on 436 flotation which justifies further investigations. Also, an experimental study of the flotation on 437 the bath-metal interface has not been realized yet, future efforts will be needed to overcome 438 the experimental difficulties.

\section{Acknowledgements}

440 The authors would like to express their gratitude to Rio Tinto Aluminium and the Natural

441 Sciences and Engineering Research Council of Canada for their financial support.

\section{Bibliography}

444 [1] C. Kaszas, L. Kiss, S. Guerard and J.-F. Bilodeau: TMS Light Metals, 2015, pp. 639-644

445 [2] P. Singh and D. D. Joseph: Fluid Mechanics, 2005, vol. 530, pp. 31-80

446 [3] A. Solheim and S. Rolseth: TMS Light Metals, 2001, pp. 469-474

447 [4] Pushpendra Singh, Daniel D. Josep and Nadine Aubry: Soft Matter, 2010, no. 6, pp.

$448 \quad 4310-4325$

449 [5] Victor. M. Starov, Manuel G. Velarde, Clayton J. Radke: Wetting and Spreading

450 Dynamics, Surfactant Science Series vol 138, $1^{\text {st }}$ ed., CRC Press, 2007.

451 [6] J. Thonstad and Y.-X. Liu: TMS Light Metals, 1981, pp. 303-312

452 [7] D. Bunyak: Mining History Journal, 2000, pp. 35-45

453 [8] B. Kowalczuk Przemyslaw and J. Drzymala: Colloids and Surfaces A: Physicochem. Eng. 454 Aspects, 2012, 393, pp. 81-85.

455 [9] Daniel Chipfunhu, Massimiliano Zanin, Stephen Grano: Minerals Engineering, 2011, 24, pp. 456 50-57. 
457 [10] P. B. Kowalczuk, O. Sahbaz and J. Drzymala: Minerals Engineering, 2011, 24, pp. 766458771.

459 [11] David I.Walker: Alumina in aluminium smelting and its behaviour after addition to 460 cryolite-based electrolytes, PhD thesis, 1993, University of Toronto

461 [12] M. Kucharik and R. Vasiljev: Z. Naturforsch, 2006, no. 61a, pp. $389-398$

462 [13] A. Solheim: TMS Light Metals, 2002, pp. 225-230

463 [14] J. Thonsta, Pavel Fellner, Geir Martin Haarberg, Jan Hives, Halvor Kvande, Asmund 464 Sterten: Aluminium Electrolysis - Fundamentals of the Hall-Heroult Process, $3^{\text {rd }}$ ed., Aluminium465 Verlag, Dusseldorf, 2001, pp. 87-114

466 [15] K. Grjotheim and H. Kvande, Introduction to Aluminium Electrolysis, $2^{\text {nd }}$ ed., Aluminium467 Verlag, Dusseldorf, 1993, pp. 60-85

468 [16] V. Danek, O. Patarak and T. Ostvold: Canadian Metallurgical Quarterly, 1995, vol. 34, no. 469 2, pp. 129-133

470 [17] M. Vermot Des Roches, Modèles de tension de surface pour les sels fondus et les 471 métaux liquides appliqués au procédé Hall-Héroult, master thesis, 2018, University of Montreal

472 [18] T. Utigard and J. M. Toguri, METALLURGICAL TRANSACTIONS B, 1985, Vol 16B, p 333-338

473 [19] J. Alari, L. Kiss, S. Poncsak, T. Roger: The Encyclopaedia of Research on Aluminium in 474 Quebec 2018, REGAL Students' Day Aluminium Research Centre - REGAL, pp15 